\title{
HBV/HDV co-infection in the Western Brazilian Amazonia: an intriguing mutation among HDV genotype 3 carriers
}

\author{
A. Kay, ${ }^{1}$ E. Melo da Silva, ${ }^{1}$ H. Pedreira, ${ }^{2}$ S. Negreiros, ${ }^{3}$ C. Lobato, ${ }^{3}$ W. Braga, ${ }^{4}$ \\ R. Muwonge, ${ }^{5}$ P. Dény, ${ }^{1}$ M. Reis, ${ }^{2}$ F. Zoulim, ${ }^{1,6}$ C. Trepo, ${ }^{1,6}$ A. D'Oliveira Jr, ${ }^{7}$ \\ J. M. Salcedo, ${ }^{8,9}$ M. I. Schinoni ${ }^{7}$ and R. Parana ${ }^{2,7}{ }^{1}$ INSERM, U1052, Lyon, France; ${ }^{2}$ Laboratory of Pathology and \\ Molecular Biology, CPqGM/FIOCRUZ, Salvador, Brazil; ${ }^{3}$ Acre State Secretariat of Health, Rio Branco, Brazil; ${ }^{4}$ Institute of Tropical Medicine of \\ Amazonas, Manaus, Brazil; ${ }^{5}$ International Agency for Research on Cancer (IARC)/World Health Organization (WHO), Lyon, France; ${ }^{6}$ Service \\ d'Hépato-Gastroentérology, Hôpital de la Croix-Rousse, Lyon, France; ${ }^{7}$ University Hospital, Federal University of Bahia, Amazon Hepatitis Group, \\ Salvador, Brazil; ${ }^{8}$ Federal University of Rondônia, Porto Velho, Brazil; and ${ }^{9}$ FIOCRUZ-RO, Porto Velho, Brazil
}

Received October 2013; accepted for publication April 2014

SUMMARY. HDV infection still remains a serious public health problem in Amazonia. There are few data regarding the biomolecular aspects of $\mathrm{HBV} / \mathrm{HDV}$ co-infection in this region. We studied 92 patients $\mathrm{HBsAg}^{+} / \mathrm{anti}-\mathrm{HDV} \mathrm{IgG}^{+}$followed at the Hepatitis Referral Centers of Porto Velho (RO), Rio Branco and Cruzeiro do Sul (AC), Brazil, from March 2006 to March 2007 for whom the HDV and/or the HBV genotype could be determined. The HDV genotype could be determined in 90 patients, while the HBV genotypes could be positively determined in 74 . HBV subgenotype $\mathrm{F} 2$ is the most prevalent $(40.2 \%)$, followed by the subgenotypes A1 (15.2\%) and D3 (8.7\%), while 16.4\% were other subgenotypes or genotypes, $4.3 \%$ were discordant and $15.2 \%$ were unamplifiable. Surprisingly, $\mathrm{HDV}$ genotype 3 (HDV-3) was found in all of the HBV/ HDV-infected patients that could be genotyped for HDV, confirming that HDV-3 can associate with non-F HBV genotypes. However, a HDV-3 mutant was found in 29.3\% of patients and was more frequently associated with non-F HBV genotypes $(P<0.001)$ than were nonmutant strains, suggesting that the mutation may facilitate association of HDV-3 with non-F HBV genotypes.

Keywords: HBV/HDV co-infection, HBV/HDV genotypes, HDV genotype 3, mutant HDV genotype 3.

\section{INTRODUCTION}

It is estimated that more than 10 million people are infected with hepatitis D virus (HDV) worldwide. Most hyperendemic areas are located in developing countries, such as Brazilian Amazonia. Although considered a vanishing disease in developed countries, hepatitis D is still a priority research field in many countries, including those of Latin America that are contained within the Amazonian Basin. $\mathrm{HDV}$ infection is very particular in this area, not only for the presence of HDV genotype 3 (HDV-3, previously designated genotype III) but also for the predominant Amerindian ethnicity and epidemiological aspects [1]. Molecular epidemiological studies have demonstrated that HBV genotype $\mathrm{F}$ and HDV genotype 3 circulate in this area,

Abbreviations: HDV, hepatitis D virus; HDV-3, HDV genotype 3.

Correspondence: Alan Kay, INSERM U1052, 151 cours Albert Thomas, 69003 Lyon, France.

E-mail: alan.campbell.kay@gmail.com particularly among isolated communities, including Amerindian populations $[1,2]$.

In this region, a peculiar form of fulminant hepatitis called Labrea Black Fever has been reported since the 1930s [3]. Some authors suggest that the co-infection by these Amazonian HBV/HDV genotypes is related to this severe disease $[1,4]$, while other suggest that infection by HAV, HBV and HCV may also trigger fulminant hepatitis. A similar disease was also reported in the African equatorial forest, where it also affected young individuals and presented high lethality rates, similar to the cases reported in the Amazonian region [5]. Intriguingly, both diseases have implicated HDV infection and shared the same histopathologic peculiarities.

Although HDV/HBV co-infection is considered hyperendemic in Amazonia, there is no clear documentation about the interplay of HBV/HDV genotypes in this area. This study aims to contribute to the knowledge of the HBV/ HDV co-infection and the genotypes predominant in Brazil as well as their distribution and the factors with which they are associated. 


\section{MATERIALS AND METHODS}

\section{Patients}

Of $92 \mathrm{HBV} / \mathrm{HDV}$ patients co-infected and followed at the Hepatitis Referral Centers of Porto Velho, Rio Branco and Cruzeiro do Sul from March 2006 to March 2007 were enrolled in this study.

Informed consent was obtained from each patient. The protocol was approved by the Ethics Committees of HUPES, Salvador and University Hospital, Rio Branco.

\section{HBV DNA and HDV RNA extraction}

Total nucleic acids were extracted from $200 \mu \mathrm{L}$ of serum using the High Pure Viral Nucleic Acid kit (Roche Applied Science, Meylan, France) according to the manufacturer's instructions. Normal human serum was used as control for each extraction.

\section{HBV amplification and genotyping}

HBV DNA was amplified by PCR and nested PCR using specific primers (Table 1 ) in the $\mathrm{PreC} / \mathrm{C}$ region and/or the $\mathrm{S}$ gene.

PCR or nested PCR products were sent to Genome Express (Grenoble, France) for sequencing. Edited sequences were submitted to BLAST analysis, and the highest scoring complete $\mathrm{HBV}$ genome was retained. A phylogenetic tree was constructed from $>2000$ complete HBV genome sequences contained in GenBank, and HBV genotype/subgenotype clades were identified based on information in the GenBank entries and/or in original publications. This enabled identification of the HBV genotype and in most cases of the subgenotype.

\section{HDV amplification and genotyping}

HDV amplification and genotyping was performed essentially as described before [2]. Briefly, part of the HDV genome was amplified by RT-PCR and nested PCR using oligonucleotides shown in Table 1. Reaction products were Southern blotted and successively hybridized with radioactive probes specific (Table 1) for HDV genotypes 1 (HDV1) and 3 (HDV-3), with stripping of the membranes between hybridizations.

\section{RESULTS}

The patients' samples were characterized according to HBV and HDV genotypes (Table 2). For the 92 samples, 14 were HBV DNA negative even in nested PCR and could not be genotyped, while four were discordant between the $\mathrm{C}$ and $\mathrm{S}$ gene sequences. Of the $74 \mathrm{HBV}$ samples that could be positively genotyped, $42 / 74(56.8 \%)$ were genotype $\mathrm{F}$ and $32 / 74(43.2 \%)$ had non-F genotypes. The subgenotypes F2 (37/74, 50\%), A1 (14/74, 18.9\%) and D3 (8/74, $10.8 \%)$ were the most prevalent.

HDV sequences could be amplified from 90 of the 92 patients (Table 2) and were genotyped using genotype-specific hybridization. Surprisingly, all were HDV-3. However, 27 samples showed anomalous hybridization, with hybridization signals being weak compared to the amount of amplified HDV material present as shown by ethidium

Table 1 Oligonucleotides used in this study

\begin{tabular}{lll}
\hline Name & Function & Sequences/positions* $^{*}$ \\
\hline $\mathrm{C} 1+$ & HBV C gene PCR, 1st round forward & ${ }^{1824}$ TTCACCTCTGCCTAATCATC $^{1843}$ \\
$\mathrm{C} 1-$ & HBV C gene PCR, 1st round reverse & ${ }^{2473}$ TGWGTCCAAGGRATACTAAC $^{2454}$ \\
$\mathrm{C} 2+$ & HBV C gene PCR, 2nd round forward & ${ }^{1864}$ TCAAGCCTCCAAGCTGTGCC $^{1883}$ \\
$\mathrm{C} 2-$ & ${ }^{2419}$ CGGCGATTGAGAYCTTCGTC $^{2400}$ \\
$\mathrm{C}-\mathrm{seq}$ & HBV C gene PCR, 2nd round reverse & ${ }^{2395}$ AGGCGAGGGAGTTCTTCTTC $^{2376}$ \\
S56-F & HBV C gene, sequencing & ${ }^{56}$ CCTGCTGGTGGCTCCAGTTC $^{75}$ \\
S797-R1 & HBV S gene, 1st \& 2nd round forward & ${ }^{797}$ GCGGYAWAAAGGGACTCAAG $^{778}$ \\
S760-R2 & HBV S gene PCR, 1st round reverse & ${ }^{760}$ CCCCCAATACCASATCATCC $^{741}$ \\
Delta100 & HBV S gene semi-nested PCR reverse & ${ }^{306}$ TCCAGAGGACCCCTTCAGCGAAC $^{328}$ \\
Delta101b & HDV RT-PCR forward & ${ }^{1287}$ GAAGGAAGGCCCTSGAGAACAAG $^{1265}$ \\
Delta102b & HDV RT-PCR reverse & ${ }^{881}$ TGGAGATSCCATGCCGACCCGAAG $^{904}$ \\
Delta108 & HDV nested PCR forward & ${ }^{1234}$ CTCAGCAGGGARGAGGAAGAGGAAC $^{1210}$ \\
Delta109 & HDV nested PCR reverse (genotype 1) & ${ }^{1234}$ CTCAGCCRAGAGGAAGAAGAGGAAC $^{1210}$ \\
Geno1b & HDV nested PCR reverse (genotype 3) & ${ }^{997}$ GGGAAGAGTATATCCYAYGG $^{1016}$ \\
Geno3b & HDV hybridization, genotype 1 probe & ${ }^{1003}$ AACCCATACTATGGGAACTG ${ }^{1022}$ \\
\hline
\end{tabular}

*HBV oligonucleotides are numbered using the numbering system for HBV genotype A strains, with nucleotide 1 being the 4th base of the (often hypothetical) Eco R1 site. HDV oligonucleotides are numbered based on the original sequence (GenBank accession number X04451) of Wang et al. [9]. Degenerate nucleotides are identified using standard IUPAC codes. 
Table $2 \mathrm{HBV}$ and HDV genotype frequencies of all 92 patients

\begin{tabular}{|c|c|c|c|}
\hline Genotype & $N(\%)$ & Subgenotype & $N(\%)$ \\
\hline \multicolumn{4}{|l|}{ HBV } \\
\hline \multirow[t]{2}{*}{$\mathrm{F}$} & $42(45.7)$ & F1 & $5(5.4)$ \\
\hline & & $\mathrm{F} 2$ & $37(40.2)$ \\
\hline \multirow[t]{6}{*}{ Non-F } & $32(34.8)$ & A & $3(3.3)$ \\
\hline & & A1 & $14(15.2)$ \\
\hline & & A2 & $5(5.4)$ \\
\hline & & $\mathrm{C} 2$ & $1(1.1)$ \\
\hline & & D2 & $1(1.1)$ \\
\hline & & D3 & $8(8.7)$ \\
\hline \multirow[t]{3}{*}{ Discordant } & $4(4.3)$ & D3/F2 & $2(2.2)$ \\
\hline & & $\mathrm{D} / \mathrm{A} 1$ & $1(1.1)$ \\
\hline & & $\mathrm{A} 1 / \mathrm{F} 1$ & $1(1.1)$ \\
\hline Nonamplified & $14(15.2)$ & & \\
\hline \multicolumn{4}{|l|}{ HDV genotype 3} \\
\hline Wild type & $63(68.5)$ & & \\
\hline Mutant & $27(29.3)$ & & \\
\hline Nonamplified & $2(2.2)$ & & \\
\hline \multicolumn{4}{|l|}{$\mathrm{HBV} / \mathrm{HDV}$} \\
\hline $\mathrm{F} / \mathrm{wt}$ & $37(40.2)$ & & \\
\hline $\mathrm{F} / \mathrm{mut}$ & $5(5.4)$ & & \\
\hline Non-F/wt & $13(14.1)$ & & \\
\hline Non-F/mut & $17(1)$ & & \\
\hline Disc/wt & $4(63.0)$ & & \\
\hline $\mathrm{NA} / \mathrm{wt}$ & $9(14.3)$ & & \\
\hline $\mathrm{NA} / \mathrm{mut}$ & $5(18.5)$ & & \\
\hline Non-F/NA & $2(2.1)$ & & \\
\hline
\end{tabular}

wt, wild type; mut, mutant; disc, discordant; NA, nonamplified.

bromide staining (Fig. 1). Fourteen samples showing normal hybridization profiles and nine presenting anomalous profiles were sequenced (Accession numbers HG531147HG531169). All samples were HDV genotype 3, but while the samples showing a normal hybridization profile had

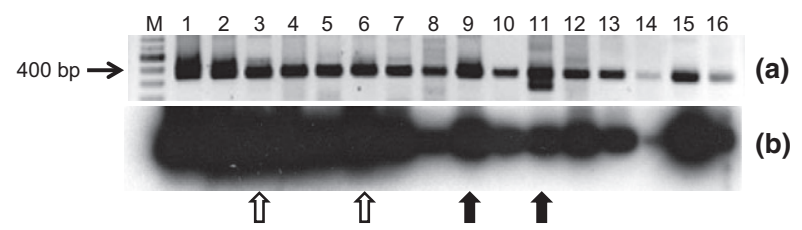

Fig. 1 Hybridization of HDV nested PCR products with the genotype 3 probe. HDV nested PCR fragments were electrophoresed on an agarose gel and stained with ethidium bromide (Panel a) before hybridization with the genotype 3 probe (Panel b). Arrows point to four samples that show approximately equally efficient amplification of HDV as shown by ethidium bromide staining. Open arrows: samples showing wild-type hybridization; full arrows: samples showing 'anomalous' hybridization. wild-type sequences, anomalous samples all had the same mutation changing the penultimate amino acid of small delta antigen (aa 193) from Phe to Tyr. This mutation occurs within the probe used for hybridization, explaining the different profiles. As the probe is short and all the anomalous samples chosen for sequencing were F193Y mutants, it is probable this mutation is responsible for the anomalous hybridization of all 27 samples. It is close to the site of RNA edition that permits the expression of large delta antigen and could potentially affect this process. However, modelling the structure of the HDV genotype 3 editing complex [6] shows that for both the wild-type sequences and the mutant sequences, the mutation site is within a non-base-pairing bulge and probably has little effect on RNA editing. This mutation is rare, F193 being highly conserved among all HDV genotypes. A search of 1166 delta antigen sequences in protein databases found only six sequences containing F193Y, three being HDV-3 isolates from Brazil [7]. This is therefore a mutation rather than a polymorphism. In our strains, the only other comparable nonsynonymous variation is N179S in 10 clones, but these are shared equally among clones with or without F193Y. Of the clones with F193Y, five could not be genotyped for HBV. Also, of the 74 samples that could be genotyped for HBV, two could not be genotyped for HDV. Therefore, only 72 samples could be used for $\mathrm{HBV} / \mathrm{HDV}$ associations. Seventy-seven per cent $(17 / 22)$ of samples with mutant HDV were co-infected with non-F HBV, while only $26 \%(13 / 50)$ of the HDV-3 wild-type samples were co-infected with non-F HBV. This was statistically significant $(P<0.001)$.

\section{DISCUSSION}

HDV-3 has only been found in Amazonia (Brazil, Peru, Venezuela). HBV genotype $\mathrm{F}$ is also found in here, and phylogenetic differences between HBV genotype $\mathrm{F}$ in different areas indicate that HBV-F infection has circulated in isolated populations for many centuries, long before European colonization. The presence now of African and European HBV genotypes can be explained by the immigration movement that occurred in Amazonia during the rubber boom of the 19th/20th century.

Some reports suggest that $\mathrm{HDV}-3$ is preferentially associated with HBV genotype $\mathrm{F}[1,4]$. In this study, we demonstrate that HDV-3, mutated or not, can also coinfect non-F genotype HBV carriers, especially those with subgenotype A1, the most frequent among non-F genotypes in this region. Almost $42 \%(30 / 72)$ of HDV-3/HBV co-infections have occurred with non-F HBV genotypes (Table 2). HDV-3/HBV-A co-infection has been reported by Gomes-Gouvëa [8] who found a high frequency of HBV-A among patients infected by HDV-3, and it has been shown that HDV-3 nucleocapsids can be packaged by non-F HBsAg. That all referral centres involved in this study were 
located in more complex urban centres could explain the diversity of HBV genotypes found here. These non-F HBV genotypes, together with HDV-1, were probably introduced into Amazonia during the rubber boom when there was extensive contact between European, Oriental and Amazonian people.

Association of HDV-3 with HBV-F may be crucial for persistence of both viruses in Amazonia. As HDV-3 seems to have co-existed, perhaps co-evolved, in Amazonia for a long time with HBV-F, it is possible that its association with non-F HBV genotypes is less efficient for HDV-3 replication. There is then a rational that the F193Y substitution described here could represent an adaptive mutation that allows HDV-3 to efficiently superinfect or co-infect non-F HBV genotypes. However, some patients with non-F $\mathrm{HBV}$ genotypes also seem to have wild-type HDV-3, meaning that HDV-3 can also associate with non-F HBV genotypes without major mutation. The role of this mutation in the evolution of HDV-3 remains to be defined, as well as its possible role in disease severity.

\section{ACKNOWLEDGEMENTS}

This work is part of a Brazilian/French cooperation programme supported by Institut National de Recherche Medical (INSERM), Agence Nationale de Recherches sur le SIDA et les hépatites virales (ANRS), Bahia's State Research Foundation (Fapesb) and Conselho Nacional de Desenvolvimento Cientifico e Tecnologico (CNPq).

\section{CONFLICT OF INTERESTS}

None of the authors has a conflict of interest relating to this study. The funding bodies did not intervene in the conduct or writing of this study.

\section{REFERENCES}

1 Casey JL, Niro GA, Engle RE et al. Hepatitis B virus (HBV)/hepatitis D virus (HDV) coinfection in outbreaks of acute hepatitis in the Peruvian Amazon basin: the roles of HDV genotype III and HBV genotype F. J Infect Dis 1996; 174(5): 920-926.

2 Parana R, Kay A, Molinet $\mathrm{F}$ et al. HDV genotypes in the Western Brazilian Amazon region: a preliminary report. Am J Trop Med Hyg 2006; 75 (3): 475-479.

3 Bensabath G, Hadler SC, Soares MC et al. Hepatitis delta virus infection and Labrea hepatitis. Prevalence and role in fulminant hepatitis in the Amazon Basin. JAMA 1987; 258(4): 479-483.
4 Nakano T, Shapiro CN, Hadler SC et al. Characterization of hepatitis D virus genotype III among Yucpa Indians in Venezuela. J Gen Virol 2001; 82(Pt 9): 2183-2189.

5 Lesbordes JL, Ravisse P, Georges AJ et al. Studies on the role of HDV in an outbreak of fulminant hepatitis in Bangui (Central African Republic). Prog Clin Biol Res 1987; 234: 451-459.

6 Linnstaedt SD, Kasprzak WK, Shapiro BA, Casey JL. The fraction of RNA that folds into the correct branched secondary structure determines hepatitis delta virus type 3 RNA editing levels. RNA 2009; 15(6): 1177-1187.

7 Gomes-Gouvea MS, Soares MC, Bensabath G et al. Hepatitis B virus and hepatitis delta virus genotypes in outbreaks of fulminant hepatitis (Labrea black fever) in the western Brazilian Amazon region. $J$ Gen Virol 2009; 90(Pt 11): 2638-2643.

8 Gomes-Gouvea MS, Pereira Soares Mdo C, Guedes de Carvalho Mello IM et al. Hepatitis D and B virus genotypes in chronically infected patients from the Eastern Amazon Basin. Acta Trop 2008; 106(3): 149-155.

9 Wang KS, Choo QL, Weiner AJ et al. Structure, sequence and expression of the hepatitis delta (delta) viral genome. Nature 1986; 323(6088): 508-514. 